\title{
Influencia del perfil del entrenador en el diseño de tareas en fútbol-base. Estudio de casos Influence of coach profile on the design of training tasks in initiation to football. A case study Francisco José Urbano-Arévalo, David Mancha-Triguero, Carlos D. Gómez-Carmona, José M. Gamonales Universidad de Extremadura (España)
}

\begin{abstract}
Resumen. Los objetivos del presente estudio fueron analizar el perfil autodefinido del entrenador de Fútbol-Base y conocer su modo de actuación durante dos mesociclos competitivos en la categoría juvenil. Se analizaron 323 tareas de entrenamiento de tres entrenadores con diferente perfil autodefinido identificado mediante los cuestionarios: i) Orientación de los Entrenadores; ii) Escala de Conocimientos y Capacidades; iii) Estilos de Decisión en el Deporte; y iv) Estilo de Planificación Deportiva. Las variables pedagógicas y organizativas durante las tareas de entrenamiento fueron clasificadas mediante la herramienta SIATE. Los estadísticos Chi cuadrado $(\div 2)$, Coeficiente Phi (öc), Test de Fisher ( $f$ ), y Residuos Tipificados Corregidos $(R T C)$ fueron empleados para identificar diferencias y grado de asociación entre variables. El perfil autodefinido del entrenador influye directamente en el diseño de las tareas con un grado de asociación moderadoalto $(\div 2=174.692-24.409 ; \ddot{o} c=0.520-0.194)$. Por tanto, su análisis es esencial para la mejora formativa del entrenador y el proceso de enseñanza-aprendizaje en el fútbol-base.
\end{abstract}

Palabras claves: Fútbol, formación, planificación, SIATE.

\begin{abstract}
The aims of the present study were to analyze the self-defined profile of initiation-to-football coaches and to evaluate their acting behaviours during two competitive mesocycles in youth football. 323 training tasks of three coaches were analyzed. Self-defined profiles were identified through the questionnaires: i) Coaches Orientation; ii) Scale of Knowledge and Capabilities; iii) Decision Styles in Sport; and iv) Sports Planning Style. The pedagogical and organizational variables during the training tasks were classified using the SIATE tool. The statistics Chi square $(\div 2)$, Phi coefficient $(\ddot{c} c)$, Fisher's exact test $(f)$, and Adjusted Standardized Residuals (ASR) were used to identify differences and association degree between variables. Coaches' self-defined profile directly influences the design of training tasks with a moderate-to-high association degree $(\div 2=174.692-24.409 ; \ddot{o} c=0.520-0.194)$. Therefore, their analysis is essential for coaches' training improvement and teaching-learning process in initiation to football.
\end{abstract}

Key words: Football, training, planning, SIATE.

\section{Introducción}

El fútbol es el deporte más popular en el mundo, practicado por personas sin importar género, edad, condiciones sociales o demográficas (Aguiar, Botelho, Lago, Maças \& Sampaio, 2012). En el fútbol al igual que en el resto de los deportes de invasión, el rendimiento depende de una serie de factores técnicos, biomecánicos, tácticos, psicológicos y fisiológicos (Stølen, Chamari, Castagna \& Wisløff, 2005), siendo el entrenamiento el único método contrastado que prepara y optimiza al deportista para la competición (Sgrò, Bracco, Pignato \& Lipoma, 2018).

El entrenamiento como instrumento de mejora debe ser sistemático, planificado, organizado y estructurado previamente por el entrenador (Ibáñez, 2008), con la finalidad de mejorar la seguridad, el orden, la variedad, la eficiencia, la mejora y la coordinación del trabajo (Giménez \& Sáenz-López, 2000), permitiendo adquirir un aprendizaje progresivo de los jugadores, siendo coherente con el proceso formativo (SáenzLópez, Ibáñez, Giménez, Sierra \& Sánchez, 2005; Sáenz-López, Jiménez, Giménez \& Ibáñez, 2007; Cañadas, Ibáñez, Feu, García-Rubio \& Parejo, 2011). Por tanto, la planificación, el desarrollo y el control del entrenamiento son responsabilidad del entrenador (Feu \& Ibáñez, 2001; Feu, Ibáñez, Lorenzo, Jiménez \& Cañadas, 2012). Esto confirma que cuanto mayor es su experiencia, mayor es el número de recursos que disponen para afrontar el diseño de los entrenamientos (Ibáñez, Feu \& Cañadas, 2016), puesto que influye directamente en el diseño de las tareas, viéndose afectada las demandas de carga interna y externa de los deportistas (Gómez-

Fecha recepción: 01-10-19. Fecha de aceptación: 14-01-20

David Mancha Triguero

dmanchat@alumnos.unex.es
Carmona, Gamonales, Feu \& Ibáñez, 2019; Gómez-Carmona, Gamonales, Pino-Ortega \& Ibáñez, 2018), o de los alumnos durante las clases de Educación Física (García-Ceberino, Antúnez, Feu \& Ibáñez, 2020).

En esta línea, la planificación de las tareas de entrenamiento en los deportes de equipo, ha evolucionado en los últimos años siendo uno de los recursos más utilizados el diseño de juegos modificados, puesto que es una herramienta para el desarrollo tanto del aspecto físico (Hill-Haas, Coutts, Rowsell \& Dawson, 2009), como del técnico-táctico (Gómez-Carmona et al., 2018), con gran interés científico y deportivo (Cañadas, Ibáñez, García, Parejo \& Feu, 2013; Gracia, García-Rubio, Cañadas \& Ibáñez, 2014; Hernández, Ortega, Mayordomo \& Palao, 2016). Por tanto, analizar la figura del entrenador y metodología empleada, aporta información relevante sobre el diseño realizado y la formación del deportista (Milistetd, Trudel, Mesquita \& Do Nascimento, 2014; Cassidy, Jones \& Potrac, 2004), puesto que es el máximo responsable en el proceso de formación (Feu \& Ibáñez, 2001). El diseño del proceso formativo dirigido por el entrenador, así como la metodología empleada, es fruto de la formación que ha recibido, las experiencias previas, o la capacidad de innovación (Clemente, Martins \& Mendes, 2015).

En relación con los métodos de enseñanza, la planificación del entrenador puede ser abordada desde una metodología tradicional o desde una metodología más comprensiva. La selección de una de ellas influirá en el proceso formativo dando lugar a una mayor especificidad con el objetivo de aportar a los jugadores una mayor riqueza técnico-táctica y que puedan construir su propio aprendizaje autónomo (González-Espinosa, Ibáñez, Feu \& Galatti, 2017). Por este motivo, tener un mayor conocimiento sobre el entrenador, puede aportar información relevante sobre su planificación y desarrollo del aprendizaje de los deportistas (Gamonales, 
Gómez-Carmona, León, García-Santos, Gamero-Portillo \& Muñoz-Jiménez, 2019; Gómez-Carmona, García-Rubio, Muñoz-Jiménez \& Gamonales, 2018). Para conocer de manera fiable al entrenador, se crearon los perfiles de entrenador. Estos perfiles catalogan al entrenador en función de diferentes áreas en 6 posibles opciones: (a) entrenador tradicional, (b) entrenador tecnológico, (c) entrenador innovador/ creativo, (d) entrenador dialogador/comunicador, (e) entrenador colaborador y (f) entrenador crítico (Ibáñez, 1996), aunque estos perfiles no deben considerase como puros o rígidos (Gamonales, Gómez-Carmona, Córdoba-Caro \& Ibáñez, 2019). Esto es debido a que el entrenador puede posicionarse entre varios perfiles e incluso puede evolucionar en función del contexto, la formación adquirida o la experiencia (Ibáñez \& Feu, 2000; López-Muñiz, Jaenes, VázquezCano \& López-Meneses, 2019; López-Muñiz, Vázquez-Cano, Jaenes \& López-Meneses, 2018). Por tanto, los entrenadores tienden a desarrollar un perfil autodefinido mixto (Feu, Ibáñez, Graçca \& Sampaio, 2007), y concretamente, en el fútbol-base deben dominar un amplio repertorio de contenidos, así como tener una gran capacidad de resolución de problemas (Gamonales, Gómez-Carmona, Córdoba-Caro et al., 2019).

Tras la revisión previa de la literatura, se encuentra la existencia de un déficit respecto a las investigaciones relacionadas con la metodología del entrenamiento y el diseño del proceso formativo de deporte base. Por ello, este estudio tiene como objetivos: (i) caracterizar el perfil autodefinido de tres entrenadores de fútbol base en la categoría juvenil; (ii) describir las variables pedagógicas que definen las tareas de entrenamiento diseñadas durante dos mesociclos en el período competitivo, así como (iii) analizar las diferencias entre el perfil autodefinido del entrenador y las características de las tareas diseñadas durante los entrenamientos.

\begin{tabular}{|c|c|c|}
\hline Variable & Núcleo categorial & Rango de apertura \\
\hline \multirow{4}{*}{ Contextuales } & Experiencia como jugador & A cada año de experiencia, se la signa un número \\
\hline & Experiencia como entrenador & A cada año de experiencia, se la signa un número \\
\hline & Formación académica & ESO, Bachillerato, FP medio, FP superior, Estudios superiores, Doctorado \\
\hline & Formación federativa & Ninguna, Monitor, Entrenador, Técnico Deportivo \\
\hline \multirow{8}{*}{ Pedagógicas } & Situación de Juego (SJ) & $\begin{array}{l}1 \times 0,1 \times 1,2 \times 0,2 \times 1,2 \times 2,2 \times 3,3 \times 0,3 \times 1,3 \times 2,3 \times 3,4 \times 0,4 \times 1,4 \times 2,4 \times 3,4 \times 4,5 \times 0 \\
5 \times 1,5 \times 2,5 \times 3,5 \times 4,5 \times 5,6 \times 2,6 \times 3,6 \times 4,6 \times 5,6 \times 6,7 \times 2,7 \times 3,7 \times 6,7 \times 7,8 \times 4,8 \times 5 \\
8 \times 6,8 \times 7,8 \times 8,9 \times 1,9 \times 9,10 \times 8,10 \times 10, \text { NxN, Combinado }\end{array}$ \\
\hline & Presencia de Portero (POR) & Con portero y Sin portero \\
\hline & Fase de Juego (FJ) & $\begin{array}{l}\text { Ataque, Defensa, Mixta, Calentamiento, Preparación Física, Recuperación, } \\
\text { Evaluación }\end{array}$ \\
\hline & Tipo de contenido (CONT-G) & $\begin{array}{l}\text { CTTIA, GTTIA, GTTID, CTTGA, CTTGD, GTTGA, CTTCA, CTTCD, GTTCA, } \\
\text { Pase, Estiramientos, Calentamiento, Preparación Física, Partido entrenamiento }\end{array}$ \\
\hline & Contenido específico (CONT-E) & $\begin{array}{l}\text { Control, Conducción, Conducción de balón, Regate, Lanzamiento portería, } \\
\text { Vigilancia individual, Pases, Pases en largo, Centros laterales, Desmarques, } \\
\text { Ataques, Ataque de inicio estratégico, Ataques por recuperación de balón en juego, } \\
\text { Conservación balón-control del juego, Cambios de orientación, Velocidad en el } \\
\text { juego, Velocidad en el juego exterior o aperturas, Progresión en el juego, } \\
\text { Progresión en el juego buscando amplitud, Acciones combinadas, Ataque por } \\
\text { recuperación de balón en juego más contraataque, Basculaciones defensivas, } \\
\text { Acciones defensivas combinadas, Repliegue defensivo más evitar segundas } \\
\text { jugadas, Temporalizaciones más presión colectiva, Preparación física: velocidad, } \\
\text { Preparación fisica: resistencia, Preparación fisica: fuerza, Preparación física: } \\
\text { flexibilidad, Preparación física: combinada, Estrategia Saque de esquina, Trabajo } \\
\text { específico de portero }\end{array}$ \\
\hline & Medio de Iniciación (MIE) & $\begin{array}{l}\text { Ejercicio de aplicación simple, Ejercicio de aplicación compleja, Juego simple e } \\
\text { inespecífico, Juego simple y específico, Juego complejo e inespecífico, Juego } \\
\text { complejo y específico, Predeporte/Deporte adaptado, Deporte, Competición. }\end{array}$ \\
\hline & Línea de juego (LJ) & Portero, Central, Lateral, Medio, Extremo, Delantero, Equipo \\
\hline & Nivel de Oposición (NO) & $\begin{array}{l}\text { Sin oposición, Con oposición estática, Con oposición dinámica, Con oposición } \\
\text { modulada, Con posición }\end{array}$ \\
\hline \multirow{3}{*}{ Organizativas } & Tiempo total & Registro según la duración de las tareas por segundo \\
\hline & $\begin{array}{l}\text { Tiempo explicación } \\
\text { Tiempo útil }\end{array}$ & Registro según la duración de las tareas por segundo \\
\hline & Tiempo útil & Registro según la duración de las tareas por segundo \\
\hline
\end{tabular}

\section{Muestra}

Se analizaron todas las tareas de entrenamiento $(n=323)$ diseñadas y desarrolladas por tres entrenadores durante los meses de Octubre y Noviembre de 2018. Las características de los entrenadores participantes se muestran en la tabla 1.

\section{Variables $\boldsymbol{e}$ instrumentos}

En la presente investigación, se analizaron dos aspectos fundamentales dentro del proceso de enseñanza-aprendizaje en el contexto deportivo: el perfil autodefinido del entrenador, y las características de las tareas de entrenamiento, siendo empleada como variable dependiente el Perfil autodefinido de los entrenadores, y como variables independientes las características de las tareas: Variables contextuales, Variables pedagógicas y Variables organizativas (Tabla 2).

Herramientas para el análisis del perfil autodefinido del entrenador

\section{- Cuestionario de Orientación de los Entrenadores}

\section{Método}

\section{Diseño}

Este trabajo se posiciona dentro de los estudios empíricos con una metodología cuantitativa. Estudio descriptivo mediante un código observacional diseñado con anterioridad al comienzo de dicha investigación, natural, pues estudia una variable independiente, la cual no puede ser intervenida por el investigador. Además, es un estudio retrospectivo pues únicamente se analizan la evolución de las variables, así como la relación que existe entre ellas (Montero \& León, 2007).

\begin{tabular}{|c|c|c|c|c|c|}
\hline Entrenador & $\begin{array}{c}\text { Experiencia } \\
\text { jugador }\end{array}$ & $\begin{array}{l}\text { Experiencia } \\
\text { entrenador }\end{array}$ & $\begin{array}{l}\text { Formación } \\
\text { académica }\end{array}$ & $\begin{array}{l}\text { Formación } \\
\text { federativa }\end{array}$ & $\begin{array}{c}\text { Tareas } \\
\text { diseñadas }\end{array}$ \\
\hline $\begin{array}{c}\text { Juvenil } \\
\text { Nacional }\end{array}$ & 16 años & 16 años & FP. Superior & Entrenador & 140 \\
\hline $\begin{array}{l}\text { Primera } \\
\text { Juvenil }\end{array}$ & 26 años & 9 años & $\begin{array}{l}\text { Dr. Ciencias del } \\
\text { deporte }\end{array}$ & Entrenador & 89 \\
\hline $\begin{array}{c}\text { Segunda } \\
\text { Juvenil }\end{array}$ & 14 años & 16 años & FP. Superior & $\begin{array}{l}\text { Sin formación } \\
\text { federativa }\end{array}$ & 94 \\
\hline
\end{tabular}

(en adelante, COQ) (Feu, Ibáñez, Graçca et al., 2007): Este instrumento tiene por objetivo analizar las dimensiones que configuran los diferentes perfiles del entrenador. El cuestionario está compuesto por 46 ítems. Tras la evaluación, se identifica la orientación del entrenador en 6 tipologías: tradicional, tecnológico, innovador, colaborador, dialogador y crítico.

- Escala de Conocimientos y Capacidades (en adelante, ECC) (Feu et al., 2012): Se diseño para determinar la procedencia de los conocimientos y capacidades empleados por el entrenador. Está compuesta por 15 ítems. A partir de esta, se determinan tres procedencias de conocimiento: formación académica, formación de jugador reconvertido y formación autodidacta.

- Cuestionario de los Estilos de Decisión en el Deporte (en adelante, EDD) (Feu, Ibáñez \& Gózalo, 2007): Tiene como objetivo evaluar el estilo de toma de decisión del entrenador. Está compuesto por 12 ítems a partir de las siguientes 
dimensiones: decisiones en los objetivos, decisiones en el entrenamiento, decisiones en la disciplina y decisiones en el desarrollo de las actividades. A partir de estas dimensiones, identifica tres estilos de decisión: autoritario, permisivo y democrático.

- Cuestionario de los Estilos de Planificación Deportiva (adelante, EPD) (Feu et al., 2007): Identifica el estilo de planificación en el diseño de las tareas de entrenamiento. Está compuesto por 15 ítems a partir de las siguientes dimensiones: planificación de las sesiones, planificación de los ejercicios, planificación de los objetivos, planificación general y planificación de la metodología. A partir de estas dimensiones, identifica tres estilos de planificación: flexible, rígido e improvisador.

Herramienta para la caracterización de las variables que definen las tareas de entrenamiento

Para la categorización de las tareas de entrenamiento se empleó la herramienta denominada Sistema Integral para el Análisis de las Tareas de Entrenamiento (SIATE) (Ibáñez et al., 2016). El registro y control de la carga en el deporte base puede realizarse de diferentes modos, dependiendo del instrumental (Mancha-Triguero, García-Ceberino, Antúnez \& García-Rubio, 2018), y los recursos económicos disponibles. Por tanto, esta herramienta fue seleccionada debido a ser gratuita y objetiva (Ibáñez et al., 2016), así como puede aplicarse en el contexto deportivo y escolar (Feu, García-Rubio, Gamero \& Ibáñez, 2019; Gamero, García-Ceberino, Reina, Feu \& Ibáñez, 2020). Las variables analizadas han sido utilizadas previamente en estudios relacionados con el fútbol formativo (Gamonales, Gómez-Carmona, Córdoba-Caro et al., 2019; Gamonales, Gómez-Carmona, León et al., 2019; GómezCarmona et al., 2018).

\section{Procedimiento}

Para conocer el perfil autodefinido y el modo de actuación de los entrenadores analizados, el procedimiento utilizado estuvo formado por varias fases. En primer lugar, los entrenadores fueron informados de las características del estudio, y su papel fundamental en la investigación, firmando un consentimiento informado antes del inicio de la recogida de los datos. A continuación, se les facilitó los diferentes cuestionarios, a través de un formulario de Google, en el cual una vez contestado, se exportaron los datos a una hoja Excel con la finalidad de analizar el perfil autodefinido de cada entrenador.

Posteriormente, se realizó el proceso de observación y la recogida de datos en cada una de las sesiones de entrenamiento. Para ello, se utilizó un procedimiento similar a los existentes en la literatura científica, donde el observador realizó un proceso de entrenamiento, para después evaluar su fiabilidad respecto a los datos recogidos, mediante dos registros de las mismas observaciones en diferentes días. Se utilizó un procedimiento estadístico adaptado del coeficiente Kappa (Cohen, 1960), denominado Multirater Kappa Free (Randolph, 2005), que indica el nivel de concordancia del observador. Tras realizar, el procedimiento de fiabilidad, el observador estaba en condiciones óptimas para categorizar las tareas de entrenamiento a través de la herramienta SIATE. El promedio de valor de Kappa de confiabilidad inter-obser- vador fue 0.92, Casi Perfecto, según Landis \& Koch (1977). Por último, los datos obtenidos por ambas herramientas fueron importados en una única base de datos en formato Excel, para posteriormente, hacer el análisis de datos final mediante el programa estadístico SPSS.

\section{Análisis estadístico}

En primer lugar, se realizó un análisis descriptivo, a través de la media de cada uno de los factores del perfil autodefinido de entrenador. Para ello, fue clave interpretar los resultados proporcionados en la Hoja de Cálculo a través de los gráficos radiales de los cuestionarios COQ, ECC, EDD y EPD. Posteriormente, para conocer el modo de actuación de los entrenadores en Fútbol-Base, se realizó un análisis descriptivo (frecuencias y porcentajes) de las variables pedagógicas que definen las tareas de entrenamiento.

Para analizar las diferencias en función del perfil autodefinido del entrenador con respecto a las características de las tareas de entrenamiento que estos diseñaban se empleó Chi cuadrado $\left(\stackrel{\div^{*}}{)}\right.$ (Newell, Aitchison \& Grant, 2014). Para identificar el nivel de asociación utilizado para las variables significativas a través del Coeficiente Phi (öc), se utilizaron los siguientes valores de Crewson (2006): (a) Pequeño (<0.100), (b) Bajo (0.100-.299), Moderado (0.300-0.499), (d) Alto $(>0.500)$. Del mismo modo, se realizó la prueba exacta del Test de Fisher ( $f$ ) debido a que el valor $p$ de Fisher es exacto para todos los tamaños de la muestra, mientras que los resultados de la prueba de $\div$ pueden ser inexactos cuando los conteos de celda son demasiado pequeños (De la FuenteFernaìndez, 2016). En este estudio, existen conteos muy bajos en diversas categorías de las variables pedagógicas. Por último, la interpretación del grado de asociación entre las variables se realizó mediante los Residuos Tipificados Corregidos (RTC) de las tablas de contingencia (Field, 2009) resultantes de relacionar las variables independientes del estudio (Variables pedagógicas) entre la variable de estudio (Perfil de entrenador).

\section{Resultados}

\section{Perfil autodefinido del entrenador}

En la Figura 1, se presenta el Perfil autodefinido del entrenador en las 4 características analizadas: a) Orientación, b) Conocimientos y capacidades, c) Estilos de decisión y d) Estilos de planificación deportiva. Respecto a la orientación del entrenador (Figura 1a), se encuentra que el entrenador de Segunda Juvenil tiene bajos valores en todas las dimensiones, en especial en el apartado tecnológico. Por el contrario, los técnicos de Primera Juvenil y Juvenil Nacional presentan altos valores en todas las dimensiones, mostrando el técnico de Primera Juvenil un perfil Tecnológico/ Dialogador y el entrenador de Juvenil Nacional presenta un perfil Crítico/Colaborativo. En cuanto a la Formación del entrenador se muestra que los entrenadores de las distintas divisiones presentan un perfil académico y de autoformación. Además, cabe destacar que el técnico de Juvenil Nacional presenta valores respecto a la dimensión Jugador Reconvertido (Figura 1b). Respecto a la Toma de decisiones de los entrenadores, se observa como el entrenador de Segunda Juvenil presenta un estilo Democrático/ 

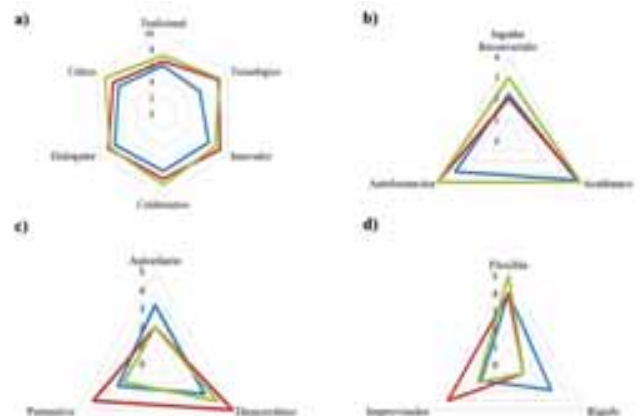

d)

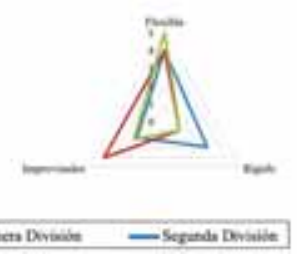

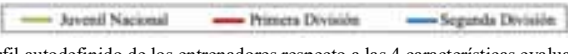

Figura 1. Perfil autodefinido de los entrenadores respecto a las 4 características evaluadas: a) Orientación, b) Conocimientos y capacidades, c) Estilos de decisión y d) Estilos de planificación deportiva.

Autoritario. Los técnicos de Primera Juvenil y Juvenil Nacional coinciden mostrando ambos un carácter Democrático, siendo el técnico de Segunda Juvenil más Permisivo a excepción del segundo (Figura 1c). Finalmente, en cuanto a los estilos de planificación deportiva, el entrenador de Segunda Juvenil presenta un estilo de planificación Rígido, el entrenador de Primera Juvenil manifiesta un estilo Improvisador, y el entrenador de Juvenil Nacional un perfil de planificación Flexible (Figura 1d).

Modo de actuación del entrenador: Variables organizativas en las tareas de entrenamiento.

En primer lugar, la tabla 3 muestra las variables organizativas de las tareas registradas en la presente investigación. Se encuentra que el entrenador de Primera Juvenil presenta un mayor Tiempo útil (92.86\%), y un menor Tiempo de explicación de las tareas (7.13\%), mientras que el entrenador de Juvenil Nacional presenta un menor Tiempo útil (88.85\%), y un mayor Tiempo de explicación de las tareas $(11.20 \%)$.

Tabla 3 .

\begin{tabular}{|c|c|c|c|c|c|}
\hline \multirow[b]{2}{*}{ Entrenador } & & \multirow[b]{2}{*}{ Tareas } & \multicolumn{3}{|c|}{ Tipos de tiempo } \\
\hline & & & Tiempo total & $\begin{array}{c}\text { Tiempo } \\
\text { explicación }\end{array}$ & Tiempo útil \\
\hline \multirow{4}{*}{ Juvenil Nacional } & \multirow{4}{*}{94} & Media $\phi \mathrm{DE}$ & $773.71 \oplus 472.87$ & $86.63 \$ 69.15$ & 687.45 \& 457.09 \\
\hline & & Máximo & 3600 & 300 & 3600 \\
\hline & & Mínimo & 210 & 0 & 180 \\
\hline & & $\%$ & 100 & 11.20 & 88.85 \\
\hline \multirow{4}{*}{ Primera Juvenil } & \multirow{4}{*}{89} & Media $\phi D E$ & $682.36 \phi 611.08$ & $48.65 \phi 75.94$ & $633.71 \notin 569.86$ \\
\hline & & Máximo & 33360 & 600 & 3120 \\
\hline & & Mínimo & 140 & 0 & 120 \\
\hline & & $\%$ & 100 & 7.13 & 92.86 \\
\hline \multirow{4}{*}{ Segunda Juvenil } & \multirow{4}{*}{140} & Media $\phi D E$ & 854.17 \& 518.01 & $84.06 \$ 87.66$ & $770.52 \notin 482.31$ \\
\hline & & Máximo & 3000 & 690 & 2700 \\
\hline & & Mínimo & 120 & 0 & 120 \\
\hline & & $\%$ & 100 & 9.84 & 90.21 \\
\hline \multirow{4}{*}{ Total casos } & \multirow{4}{*}{323} & Media $\notin D E$ & $783.41 \$ 536.19$ & $75.05 \phi 80.88$ & 708.65 \& 502.85 \\
\hline & & Máximo & 3600 & 690 & 3600 \\
\hline & & Mínimo & 120 & 0 & 120 \\
\hline & & $\%$ & 100 & 9.58 & 90.46 \\
\hline
\end{tabular}

DE: Desviación estándar

Modo de actuación del entrenador: Variables pedagógicas en las tareas de entrenamiento

El modo de actuación de los entrenadores en FútbolBase con respecto al diseño de las variables pedagógicas de

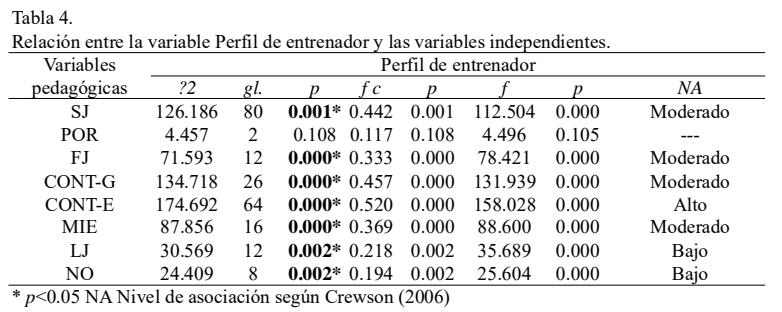

las tareas de entrenamiento y el grado de asociación respecto al perfil autodefinido se muestra en la Tabla 4. Se encuen-

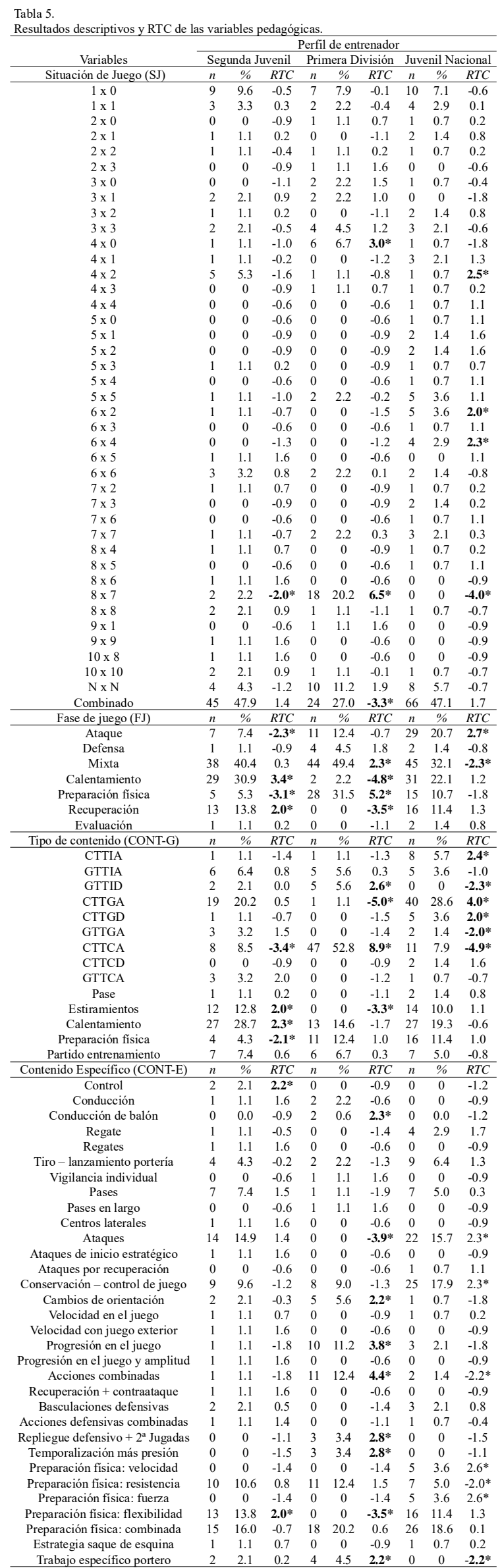




\begin{tabular}{|c|c|c|c|c|c|c|c|c|c|}
\hline Medio de iniciación entrenamiento & $n$ & $\%$ & $R T C$ & $n$ & $\%$ & $R T C$ & $n$ & $\%$ & $R T C$ \\
\hline Ejercicio de aplicación simple & 40 & 42.6 & 1.7 & 15 & 16.9 & $-4.3^{*}$ & 60 & 40.2 & $2.4^{*}$ \\
\hline Ejercicio de aplicación compleja & 6 & 6.4 & -0.1 & 9 & 10.1 & 1.6 & 6 & 4.3 & -1.4 \\
\hline Juego simple inespecífico & 7 & 7.4 & 0.5 & 0 & 0 & $-2.4 *$ & 7 & 5.0 & 1.8 \\
\hline Juego simple específico & 10 & 10.6 & 0.0 & 0 & 0 & $-3.8^{*}$ & 0 & 0 & $3.4^{*}$ \\
\hline Juego complejo inespecífico & 0 & 0 & -0.9 & 1 & 1.1 & 0.7 & 1 & 0.7 & 0.2 \\
\hline Juego complejo específico & 10 & 10.6 & $-2.4 *$ & 45 & 50.6 & $6.5 *$ & 26 & 18.6 & $-3.8 *$ \\
\hline Pre-deporte / deporte adaptado & 0 & 0 & -1.8 & 4 & 4.4 & $3.3^{*}$ & 0 & 0 & -1.3 \\
\hline Deporte & 17 & 18.1 & $2.1^{*}$ & 9 & 10.1 & -0.7 & 13 & 9.3 & -1.3 \\
\hline Competición & 4 & 4.3 & 0.1 & 6 & 6.7 & 1.5 & 3 & 2.1 & -1.5 \\
\hline Línea de juego (LJ) & $n$ & $\%$ & RTC & $n$ & $\%$ & RTC & $n$ & $\%$ & RTC \\
\hline Portero & 2 & 2.1 & 0.2 & 4 & 4.5 & $2.2 *$ & 0 & 0 & $-2.2 *$ \\
\hline Central & 2 & 2.1 & 0.9 & 0 & 0 & -1.2 & 2 & 1.4 & 0.3 \\
\hline Later & 1 & 1.1 & 0.7 & 0 & 0 & -0.9 & 1 & 0.7 & 0.2 \\
\hline Med & 11 & 11.7 & 0.6 & 0 & 0 & $-3.7^{*}$ & 22 & 15.7 & $2.9^{*}$ \\
\hline & 5 & 5.3 & 0.8 & 6 & 6.7 & 1.5 & 2 & 1.4 & $-2.1^{*}$ \\
\hline Delantero & 4 & 4.3 & -0.4 & 2 & 2.2 & -1.4 & 10 & 7.1 & 1.6 \\
\hline Equip & 69 & 73.4 & -1.0 & 77 & 86.5 & $2.5^{*}$ & 10 & 73.6 & -1.3 \\
\hline Nivel de oposición (NO) & $n$ & $\%$ & RTC & $n$ & $\%$ & $R T C$ & $n$ & $\%$ & $R T C$ \\
\hline & 50 & 53.2 & 0.8 & 39 & 43.8 & -1.3 & 72 & 51.4 & 0.5 \\
\hline Con op & 2 & 2.1 & $2.2^{*}$ & 0 & 0 & -0.9 & 0 & 0 & -1.2 \\
\hline Con opo & 1 & 1.1 & 0.2 & 2 & 2.2 & 1.5 & 0 & 0 & -1.5 \\
\hline Con oposici & 8 & 8.5 & 0.3 & 0 & 0 & $-3.2^{*}$ & 17 & 12.1 & $2.6^{*}$ \\
\hline Con oposición & 33 & 35.1 & -1.3 & 48 & 53.9 & $2.9^{*}$ & 51 & 36.4 & -1.4 \\
\hline
\end{tabular}

tran diferencias respecto al Perfil autodefinido del entrenador en todas las Variables pedagógicas analizadas excepto en la variable Presencia de portero (POR). Las diferencias encontradas son con un nivel de asociación alto la variable Contenido específico (CONT-E), con nivel moderado las variables Situación de juego (SJ), Fase de juego (FJ), Contenido general (CONT-G), y Medio de iniciación al entrenamiento (MIE), y con nivel bajo las variables Línea de juego (LJ), y Nivel de oposición (NO).

Para la interpretación de las diferencias encontradas, se presentan los resultados descriptivos de las variables significativas en la Tabla 5, así como los Residuos Tipificados Corregidos ( $R T C$ ), con la finalidad de determinar el grado de asociación entre las variables.

\section{Discusión}

El estudio tiene como objetivos principales analizar el perfil autodefinido de los entrenadores de Fútbol-Base, en la categoría Juvenil, así como, conocer los modos de actuación a través del análisis de las tareas de entrenamiento diseñadas y desarrolladas por los propios profesionales.

Los resultados obtenidos de los cuestionarios indican que los entrenadores tienen un perfil autodefinido mixto (Feu et al., 2007). Además, dependiendo del cuestionario utilizado, los técnicos se autodefinen de distinta manera. Los resultados del análisis descriptivo e inferencial de los tres entrenadores muestran que utilizan gran variedad de variables pedagógicas en sus sesiones con la finalidad de trabajar las distintas capacidades físicas, técnicas y tácticas de los jugadores en Fútbol-Base como sucede en los estudios de Gamonales, Gómez-Carmona, Córdoba-Caro et al., (2019), y Gamonales, Gómez-Carmona, León et al., (2019). Tales diferencias, están relacionadas directamente con las variables pedagógicas propuestas en el estudio y analizadas a través de la herramienta SIATE, que proporciona gran cantidad de información relacionada con los factores que inciden en el entrenamiento deportivo de los deportes de invasión (Ibáñez et al., 2016; Gamonales, Gómez-Carmona, Córdoba-Caro et al.,2019).

\section{Perfil autodefinido del entrenador}

En cuanto al Perfil autodefinido de los entrenadores en Fútbol-Base, el entrenador de Segunda Juvenil presenta un perfil Dialogador/Colaborativo, el técnico de Primera Juvenil se muestra Innovador/Tecnológico, y el entrenador de Juvenil Nacional exhibe un perfil Crítico/Colaborativo. Por tanto, los resultados muestran que existen similitudes y diferencias entre los entrenadores analizados, presentando distintas características, y estando influenciados por variables extrínsecas e intrínsecas relacionadas con la orientación, conocimientos y capacidades, toma de decisiones y estilos de planificación deportiva de los entrenadores (Feu, Ibáñez et al., 2007; Feu, Ibáñez, Graça et al., 2007). Asimismo, los técnicos se autodefinen como académicos y autoformados, con la excepción del entrenador de Juvenil Nacional que muestra valores por encima de la media en experiencia como jugador.

Coincidiendo con varios autores (Cassidy \& Rossi, 2006; Feu et al., 2012), la formación académica y la experiencia propia como jugador es muy beneficiosa y enriquecedora para el entrenador, con la finalidad de generar perfiles de entrenadores mixtos (Feu, Ibáñez et al., 2007). Por ello, la experiencia como jugador o el propio interés por formarse, son claves para generar perfiles concretos, y polifacéticos. Pues, los entrenadores en el Fútbol-Base deben de dominar un amplio repertorio de contenidos de enseñanza-aprendizaje (Gamonales, Gómez-Carmona, Córdoba-Caro et al., 2019; Gamonales, Gómez-Carmona, León et al., 2019; GómezCarmona et al., 2019). Estas diferencias encontradas en cuanto al perfil autodefinido y las características contextuales mencionadas anteriormente, afectan al diseño del entrenamiento provocando diferencias tanto en la elección de los contenidos a trabajar, como en el planteamiento de la tarea, siendo la carga y la asimilación de la tarea diferente y pudiendo verse afectado el proceso formativo del deportista. Referente a la toma de decisiones, el entrenador de Segunda Juvenil presenta un estilo Autoritario/Democrático, el técnico de Primera Juvenil muestra un estilo Permisivo/Democrático, y el entrenador de Juvenil Nacional predomina un estilo Democrático. Los años de experiencia y la forma de gestionar el equipo son un factor influyente en el desarrollo del conocimiento en los entrenadores (Cassidy \& Rossi, 2006).

En cuanto a la planificación, las diferencias entre los técnicos son aún mayores, el entrenador de Segunda Juvenil presenta un estilo Rígido, el técnico de Primera Juvenil muestra un estilo Improvisador, mientras que en el entrenador de Juvenil Nacional predomina un estilo Flexible. Estos resultados encontrados son antagónicos a los encontrados en la literatura, debido a que diferentes autores pues afirman que no se puede improvisar en la planificación (Jones, Armour \& Potrac, 2002), puesto que la planificación debe ser un proceso sensible, detallado e individualizado. La planificación es un proceso que justifica el entrenamiento deportivo (Feu \& Ibáñez, 2001), y conlleva responsabilidades (LópezMuñiz et al., 2018). Por ello, se recomienda diseñar programaciones de contenidos estándares, y que éstas se trabajen según la etapa formativa (Gamonales, Gómez-Carmona, Córdoba-Caro et al., 2019; Gamonales, Gómez-Carmona, León et al., 2019; Gómez-Carmona et al., 2019). De este modo, se fomentará que los entrenadores con escasa experiencia o formación puedan trabajar los contenidos esenciales según la edad del deportista, y así desarrollar los objetivos planificados. 
Modo de actuación del entrenador: Variables organizativas en las tareas de entrenamiento

Hay diferencias entre los técnicos analizados en relación a las Variables organizativas en las tareas de entrenamiento. El entrenador de Primera Juvenil presenta un mayor Tiempo útil, y un menor Tiempo de explicación de las tareas, mientras que el entrenador de Juvenil Nacional presenta un menor Tiempo útil, y un mayor Tiempo de explicación de las tareas. En la literatura científica, los estudios muestran que los entrenadores formados y con amplia experiencia emplean un mayor Tiempo útil y un menor Tiempo de explicación de las tareas (Gamonales, Gómez-Carmona, León et al., 2019; Gómez-Carmona, García-Rubio et al., 2018). Al igual que sucede con los entrenadores de rugby de Andalucía, el colectivo de técnico en fútbol-base de la localidad de Cáceres, en concreto de la categoría juvenil. no realiza aprendizaje permanente de manera formal, en algunos casos motivado por la falta de interés u oferta de formación (López-Muñiz et al., 2019; López-Muñiz et al., 2018). Por ello, se recomienda realizar estudios más amplios en relación a la formación de los entrenadores de fútbol-base, y los técnicos con mayor formación deben de estar en las categorías de iniciación y perfeccionamiento deportivo.

\section{Modo de actuación del entrenador: Variables pedagó- gicas en las tareas de entrenamiento}

Respecto al Modo de actuación de los entrenadores en Fútbol-Base, hay diferencias significativas entre los técnicos analizados. Concretamente, en las variables pedagógicas SJ, FJ, CONT-G, CONT-E, MIE, LJ y NO. Sin embargo, la Presencia del portero (POR) en las tareas de entrenamiento no está influenciado por la variable Perfil del entrenador. Es decir, no existe diferencias entre los técnicos.

La variable $S J$ presenta diferencias y similitudes entre los técnicos analizados. El entrenador de Primera Juvenil tiene mayor probabilidad de realizar tareas de entrenamiento $4 \times 0$, y menor probabilidad de desarrollar tareas combinadas. El técnico de Juvenil Nacional, presenta mayor probabilidad de lo esperado de utilizar tareas $4 \times 2,6 \times 2$ y $6 \times 4$. Y respecto al entrenador de Segunda Juvenil, tiene menor probabilidad de diseñar tareas de $8 \times 7$. Sin embargo, esta situación de entrenamiento (8x7) solamente es utilizada por el entrenador de Primera Juvenil. Los entrenadores en Fútbol-Base utilizan una gran variedad de SSGs, los cuáles poseen exigencias cinemáticas y fisiológicas semejantes al juego real, y que mejoran la toma de decisiones (Gómez-Carmona, Gamonales et al., 2018). Estas adaptaciones se deben a que las situaciones reducidas están próximas a las demandas que exige la competición, $y$, además, permite a los jugadores construir su propio aprendizaje (González-Espinosa et al., 2017), puesto que los requerimientos técnico-tácticos también son menores y facilitan el aprendizaje del deportista. Estos resultados coinciden con la literatura existente en Fútbol-Base (Gamonales, Gómez-Carmona, Córdoba-Caro et al., 2019, Gamonales, Gómez-Carmona, León et al., 2019; GómezCarmona et al., 2019; Gómez-Carmona, García-Rubio et al., 2018), puesto que son tareas que están en consonancia con los nuevos modelos de juego en un equipo de fútbol. Por ello, se recomienda realizar estudios más amplios en relación con el número de entrenadores, y temporalidad de las tareas con la finalidad de proponer propuestas realistas y adecuadas a los requerimientos de los jugadores en Fútbol-Base.

Respecto a la variable FJ y su relación con el Perfil de entrenador, existen diferencias entre entrenadores. El entrenador de Segunda Juvenil plantea tareas de Calentamiento y Recuperación, y menor probabilidad de realizar tareas específicas de Preparación física. Sin embargo, el entrenador de Primera Juvenil predomina principalmente Preparación Física y Mixtas. Por el contrario, el técnico de Juvenil Nacional muestra mayor probabilidad de realizar tareas de Ataque, y menor probabilidad de desarrollar tarea de entrenamiento Mixtas. Los resultados no corroboran lo citado por Gamonales, Gómez-Carmona, Córdoba-Caro et al., (2019), o Gómez-Carmona, García-Rubio et al., (2018) que afirman que en Fútbol-Base, en la categoría Juvenil, los entrenadores diseñan tareas mixtas caracterizadas por continuos cambios técnico-tácticos entre las fases de juego de defensa y ataque. Por ello, las tareas de entrenamiento tienen que evolucionar en la complejidad, en el número de jugadores o en la toma de decisiones por parte de los jugadores (Cañadas et al., 2011; Cañadas et al., 2013). Los entrenadores analizados enfocan las tareas a mejorar determinados aspectos de forma específica del equipo según los requerimientos de participación en la Liga Extremeña de Fútbol, en lugar de enfocar las propuestas al desarrollo de los valores a través de la participación deportiva (Maestre, Garcés, Ortín \& Hidalgo, 2019). Por tanto, los entrenadores deben diseñar tareas de entrenamiento donde se prime el trabajo en equipo y la realización de actividades de forma global y colaborativa, semejante a las características que un jugador se puede encontrar en situación de juego.

Existen diferencias significativas entre entrenadores en relación a la variable CONT-G. El entrenador de Juvenil Nacional diseña y desarrolla tareas de CTTIA/CTTGA, con mayor que probabilidad que tareas de GTTID. Sin embargo, el técnico de Primera Juvenil, presenta una menor probabilidad de realizar Estiramientos en sus entrenamientos. Y el entrenador de Segunda Juvenil, tienen una mayor probabilidad de realizar Calentamiento y Estiramientos, y menor probabilidad de trabajar la Preparación física. Además, destacar el contenido CTTCA, con mayor probabilidad de utilización en sus tareas por el técnico de Primera Juvenil, en contraposición con el resto de entrenadores. Por ello, el diseño de las tareas de entrenamiento en el Fútbol-Base, se debe partir del patrón principal que es la realidad de la competición (Casamichana et al., 2013), y la idoneidad del espacio para desarrollar los entrenamientos. Por ello, sería recomendable analizar varias temporadas, e incluso estudiar la progresión de los diferentes factores que influyen en las variables pedagógicas y el proceso formativo del deportista.

Existen diferencias entre los entrenadores en relación con la variable CONT-E. El entrenador de Segunda Juvenil tiene mayor probabilidad de los esperado de realizar tareas de Control, y Preparación física: flexibilidad. Por el contrario, el entrenador de Primera Juvenil presenta mayor probabilidad de lo esperado de diseñar tareas de Conducción de balón, Progresión en el juego, Cambio de orientación, Acciones combinadas, Repliegue defensivo + Segunda jugada, Temporalización y Trabajo específico de portero. Y el entrenador de Juvenil Nacional diseña y desarrolla tareas 
de Ataques, Conservación-Control de juego, Preparación física: Velocidad y Preparación Física: Fuerza. En la literatura científica, existen diferentes estudios relacionados con los contenidos a trabajar de forma general en las diferentes categorías de Fútbol-Base, sin embargo, no especifican claramente que contenidos específicos a trabajar por categorías (Casamichana et al., 2013), como sucede en otras modalidades deportivas como en baloncesto (Cañadas et al., 2011; Cañadas et al., 2013), o en balonmano (Feu et al., 2012). Por ello, estudios como el desarrollado son claves para determinar y conocer el posicionamiento metodológico de los entrenadores (Cañadas et al., 2011; Ibáñez et al., 2016; Maestre et al., 2019), así como, determinar hacia donde se orienta los aprendizajes con el objetivo de mejorar el nivel de entrenamiento y el proceso formativo de los deportistas.

Respecto a la variable $M I E$, existen diferencias entre los entrenadores analizados. El entrenador de Segunda Juvenil tiene mayor probabilidad de realizar Deporte, el técnico de Primera Juvenil destaca Pre-deporte, y el entrenador de Juvenil Nacional predomina en su diseño Ejercicio de aplicación simple y Juego simple específico. Además, en el entrenador de Primera Juvenil, destacan los Juego complejo específico en mayor medida que los demás entrenadores participantes del estudio. Los resultados obtenidos son diferentes a las encontrados en la literatura, incluso en investigaciones sobre la misma categoría (Gamonales, GómezCarmona, Córdoba-Caro et al., 2019; Gamonales, GómezCarmona, León et al., 2019; Gómez-Carmona et al., 2019), donde predominan las tareas de Predeporte, Deporte adaptado y SSGs. Por ello, es recomendable utilizar novedades pedagógicas acordes a las características de los jugadores y evitar el trabajo de habilidades motrices de forma aislada, pues el fútbol es un deporte impredecible en el que cada toma de decisión agrupa un conjunto de acciones que la conforman. Para ello, es necesario conocer las demandas de los jugadores durante los entrenamientos y partidos mediante dispositivos tecnológicos como son los dispositivos inerciales con la finalidad de adaptar los entrenamientos a los requerimientos de la competición y poder optimizar el rendimiento del deportista y, por ende, el del equipo.

La variable $L J$ presenta diferencias entre los entrenadores estudiados. El entrenador de Primera Juvenil utiliza en mayor probabilidad tareas enfocadas en el equipo, y el técnico de Juvenil Nacional realiza tareas centradas en trabajar la línea de medio del campo. Los estudios encontrados en la literatura existente de esta temática muestran diferencias con respecto a los resultados obtenidos en esta investigación (Gamonales, Gómez-Carmona, Córdoba-Caro et al., 2019; Gómez-Carmona, García-Rubio et al., 2018). Estas diferencias se deben principalmente a la categoría objeto de estudio, al nivel formativo de los entrenadores, así como, al nivel de exigencias de la competición. Por tanto, no es lo mismo entrenar jugadores de Segunda Juvenil, Primera Juvenil o Juvenil Nacional, puesto que los requerimientos de la competición son totalmente diferentes.

Finalmente, en cuanto a la variable $N O$, los entrenadores muestran diferencias significativas relacionadas con el Tipo de oposición que utilizan durante las tareas de entrenamiento, cada uno con distinta forma de oposición. En el FútbolBase, se diseñan tareas próximas al juego real. Por ello, los entrenadores tienen que diseñar tareas de entrenamiento para conseguir jugadores más completos (Gamonales, GómezCarmona, León et al., 2019), y debe tener un abanico amplio de recursos (Maestre et al., 2019).

\section{Conclusiones}

Los entrenadores analizados en este estudio durante dos meses competitivos tienen perfiles autodefinidos mixtos. Además, utilizan gran variedad de variables pedagógicas para diseñar sus tareas de entrenamiento, así como muestran diferencias relacionadas entre la variable Perfil de entrenador y las variables planteadas en el estudio FJ, CONT-G, CONT-E, MIE, LJ, NO y SJ.

Por ello, los técnicos de Fútbol-Base deben dominar un amplio repertorio de contenidos generales y específicos, así como, tener una gran capacidad de resolución de problemas. No existe un modelo puro de entrenador, sino que hay modelos mixtos, donde el entrenador se va adaptando en función a las características de su equipo. Por tanto, no existen dos entrenadores iguales, el entrenador puede cambiar de perfil en una misma temporada y a lo largo de los años, a la vez que va especializando y aprendiendo a lo largo de su vida profesional. Además, las tareas a diseñar y desarrollar tienen que estar en función de la edad y nivel de exigencias de la competición.

Los instrumentos COQ, ECC, EDD, EPD y SIATE utilizados en la presente investigación son gratuitos, útiles y se complementan, pues permiten determinar el perfil autodefinido y el modo de actuación de cada entrenador con la finalidad de mejorar la planificación del proceso formativo en la modalidad deportiva de aplicación. Por tanto, son herramientas que permiten registrar y analizar los diferentes factores que inciden en el entrenamiento deportivos en deportes de invasión.

Este estudio presenta diferentes limitaciones entre las que destaca tener como muestra solo tres entrenadores, y durante dos meses competitivos en Fútbol-Base. Por tanto, para investigaciones futuras se debería profundizar en los objetivos de esta investigación con la finalidad de aumentar el número de entrenadores y un mayor número de sesiones de entrenamientos. Además, el éxito de una buena investigación pasa por una adecuada planificación.

\section{Agradecimientos}

Agradecer a los tres entrenadores de fútbol base, categoría juvenil, de los equipos A.D. San Francisco y C.P. Cacereño de Cáceres (España), por la participación y disponibilidad durante el estudio. Finalmente, agradecer la subvención parcial de la presente investigación por parte de la Ayuda a los Grupos de Investigación (GR18170) de la Junta de Extremadura (Consejería de Economía e Infraestructuras); con la aportación de la Unión Europea a través de los Fondos Europeos de Desarrollo Regional (FEDER) «Una manera de hacer Europa».

\section{Referencias}

Aguiar, M., Botelho, G., Lago, C., Maças, V., \& Sampaio, J. 
(2012). A review on the effects of soccer small-sided games. Journal of Human Kinetics, 33, 103-113. https:/ /doi.org/10.2478/v10078-012-0049-x

Cañadas, M., Ibáñez, S.J., Feu, S., García-Rubio, J., \& Parejo, I. (2011). Análisis de los medios de entrenamiento en un equipo minibasket y la influencia de un programa formativo para el entrenador. Un estudio de caso. Ágora para la Educación Física y el Deporte, 13(3), 363-382.

Cañadas, M., Ibáñez, S.J., García, J., Parejo, I., \& Feu, S. (2013). Las situaciones de juego en el entrenamiento de baloncesto en categorías base. Revista Internacional de Medicina y Ciencias de la Actividad Física y del Deporte, 13(49), 41-54.

Casamichana, D., Castellano, J., Calleja-González, J., San Román, J., \& Castagna, C. (2013). Relationship between indicators of training load in soccer players. The Journal of Strength \& Conditioning Research, 27(2), 369-374. http://doi.org/10.1519/JSC.0b013e3182548af1

Cassidy, T., \& Rossi, T. (2006). Situating Learning: (Re) examining the notion of apprenticeship in coach education. International Journal of Sports Sciences \& Coaching, 1(3), 235-246. https://doi.org/10.1260/ 174795406778604591

Cassidy, T., Jones, R., \& Potrac, P. (2004). Understanding sports coaching. The social, cultural and pedagogical foundations of coaching practice. New

York: Routledge.

Clemente, F., Martins, F., \& Mendes, R. (2015). How coaches use their knowledge to develop small-side soccer games: a case study. South African Journal for Research in Sport Physical Education and Recreation, 37(1), 1-11.

Crewson, P. (2006). Applied statistics handbook. Version 1.2. Leesburg: AcaStat Software.

De la Fuente-Fernaìndez, S. (2016). Aplicaciones de la Chicuadrado: tablas de contigencias. Homogeneidad. Dependencia e independencia. Madrid: Universidad Autónoma de Madrid.

Feu, S., \& Ibáñez, S.J. (2001). La visión del entrenador en la transición a

través de deporte 2000. Revista Internacional de Medicina y Ciencias de la

Actividad Física y el Deporte, 1(3),177-187.

Feu, S., García-Rubio, J., Gamero, M.G., \& Ibáñez, S.J. (2019). Task planning for sports learning by physical education teachers in the pre-service phase. Plos One, 14, 1-18. https://doi.org/10.1371/journal.pone.0212833

Feu, S., Ibáñez, S.J., \& Gózalo, M. (2007). Propiedades psicométricas de los cuestionarios EDD y EPD para evaluar el estilo de planificación y decisión de los entrenadores. Revista de Psicología del Deporte, 16(2), 185199.

Feu, S., Ibáñez, S.J., Graça,A., \& Sampaio, J. (2007). Evaluación psicométrica del cuestionario de orientación de los entrenadores en una muestra de entrenadores españoles de balonmano. Psicothema, 19(4), 699-705.

Feu, S., Ibáñez, S.J., Lorenzo, A., Jiménez, S., \& Cañadas, M. (2012). El conocimiento profesional adquirido por el entrenador de balonmano: experiencias y formación. Revista de Psicología del Deporte, 21(1), 107-115.

Field, A. (2009). Discovering statistics using SPSS ( $3^{\text {a }}$ ed.).
London: Sage Publications Ltd.

Gamero, M.G., García-Ceberino, J.M., Reina, M., Feu, S., \& Ibáñez, S.J. (2020). Estudio de las variables pedagógicas de las tareas de baloncesto en función de las fases de juego. Retos: Nueva tendencias en educación física, deporte y recreación, 37, 556-562.

Gamonales, J.M., Gómez-Carmona, C.D., Córdoba-Caro, L., \& Ibáñez, S.J. (2019). Influencia del perfil del entrenador en el diseño de las tareas en el fútbol. Estudio de caso. Journal of Sport and Health Research, 11(1), 69-82.

Gamonales, J.M., Gómez-Carmona, C.D., León, K., GarcíaSantos, D., Gamero-Portillo, M., \& Muñoz-Jiménez, J. (2019). Análisis de las tareas de Entrenamiento en fútbolbase: diferencias entre dos meses durante el periodo competitivo en la categoría sub-19. Sportis: Revista Técnico-Científica del Deporte Escolar, Educación Física y Psicomotricidad, 5(1), 30-52. https://doi.org/10.17979/ sportis.2019.5.1.3469

García-Ceberino, J.M., Antúnez, A., Feu, S., \& Ibáñez, S.J. (2020). Quantification of internal and external load in school football according to gender and teaching methodology. International Journal of Environmental Research and Public Healt, 17(1), 1-18. https://doi.org/ 10.3390/ijerph17010344

Giménez, F.J., \& Sáenz-López, P. (2000). Aspectos teóricos y prácticos de la iniciación al baloncesto. Huelva (España): Diputación de Huelva.

Gómez-Carmona, C.D., Gamonales, J.M., Feu, S., \& Ibáñez, S.J. (2019). Estudio de la carga interna y externa a través de diferentes instrumentos. Un estudio de casos en el fútbol formativo. Sportis: Revista Técnico-Científica del Deporte Escolar, Educación Física y Psicomotricidad, 5(3), 444-468. https://doi.org/10.17979/ sportis.2019.5.3.5464

Gómez-Carmona, C.D., Gamonales, J.M., Pino-Ortega, J., \& Ibáñez, S.J. (2018). Comparative analysis of load profile between small-sided games and official matches in youth soccer players. Sports, 6(4), e173. https://doi.org/10.3390/ sports6040173

Gómez-Carmona, C.D., García-Rubio, J., Muñoz-Jiménez, J., \& Gamonales, J.M., (2018). Relación entre el medio de iniciación al entrenamiento y las variables pedagógicas que definen las tareas en el fútbol. TRANCES: Revista de Transmisión del Conocimiento Educativo y de la Salud, 10(1), 401-420.

González-Espinosa, S., Ibáñez, S.J., Feu, S., \& GalattI, L. (2017). Programas de intervención para la enseñanza deportiva en el contexto escolar, PETB y PEAB. Retos: Nueva tendencias en educación física, deporte y recreación, 31, 103-106.

Gracia, F., García-Rubio, J., Cañadas, M., \& Ibáñez, S.J. (2014). Heart rate differences in small sided games in formative basketball. E-Balonmano.com: Revista de Ciencias del Deporte, 10(1), 23-30.

Hernández, E., Ortega, E., Mayordomo, M., \& Palao, J.M. (2016). Efecto de distintos planteamientos metodológicos en clases de educación física sobre el aprendizaje de tres habilidades de voleibol. SPORT TK-Revista EuroAmericana de Ciencias del Deporte, 5(2), 59-68. https://doi.org/10.6018/264661 
Hill-Haas, S., Dawson, B., Coutts, A., \& Rowsell, G. (2009). Physiological responses and time-motion characteristics of various small-sided soccer games in youth players. Journal of Sports Sciences, 27(1), 1-8. https:// doi.org/10.1080/02640410802206857

Ibáñez, S.J. (1996). Análisis del proceso de formación del entrenador español de baloncesto. Tesis Doctoral. Universidad de Granada.

Ibáñez, S.J. (2000). La enseñanza del baloncesto dentro del contexto

educativo. Habilidad motriz, Revista de Ciencias de la Actividad Física y del Deporte, 15, 12-21.

Ibáñez, S.J., \& Feu, S. (2000). Validación de cuestionarios para clasificar los modelos de entrenadores y conocer las variables que les afectan. In:

Fuentes, J.P., \& Macías, M. Actas del I Congreso de la Asociación Española de Ciencias del Deporte, 215-225.

Ibáñez, S.J., Feu, S., \& Cañadas, M. (2016). Sistema integral para el análisis de las tareas de entrenamiento, SIATE, en deportes de invasión. E-balonmano.com: Revista de Ciencias del Deporte, 12(1), 3-30.

Jones, R.L., Armour, K., \& Potrac, P. (2002). Understanding the Coaching process: a framework for social analysis. Quest, 54(1), 34-48. https://doi.org/10.1080/ 00336297.2002 .10491765

Landis, J.R., \& Koch, G.G. (1977). Aplication of hierarchical kappa-type statistics in assessment of majority agreement among multiple observers. Biometrics, 33(2), 363-374.

López-Muñiz, G., Jaenes, J.C., Vázquez-Cano, E., \& LópezMeneses, E. (2019). Formación permanente y necesidades de formación de los entrenadores de rugby en Andalucía. Retos: Nueva tendencias en educación física, deporte y recreación, 36, 115-120.

López-Muñiz, G., Vázquez-Cano, E., Jaenes, J.C., \& LópezMeneses, E. (2018). Docencia y formación de los entrenadores de rugby en Cádiz. Apunts. Educació Física i Esports, 134(4), 84-94.

Maestre, M., Garcés, E.J., Ortín, F.J., \& Hidalgo, M.D. (2019). El perfil del entrenador excelente en fútbol base. Un estu- dio mediante grupos focales. Cuadernos de Psicología del Deporte, 18(3), 112-128.

Mancha-Triguero, D., García-Ceberino, J.M., Antúnez, A., \& García-Rubio, J.(2018). ¿Afecta la fase de juego al diseño de las tareas de un equipo de baloncesto de formación? SPORT-TK: Revista Euroamericana de Ciencias del Deporte, 7(2), 27-36. https://doi.org/10.6018/sportk.343201

Milistetd, M., Trudel, P., Mesquita, I., \& Do Nascimento, J.V. (2014). Coaching and coach education in Brazil. International Sport Coaching Journal, 1(3), 165172. https://doi.org/10.1123/iscj.2014-0103

Montero, I., \& León, O. (2007). A guide for naming research studies in Psychology. International Journal of Clinical and Health Psychology, 7(3), 847-862.

Newell, J., Aitchison, T., \& Grant, S. (2014). Statistics for sports and exercise science: a practical approach. London and New York: Routledge Taylor \& Francis Group.

Randolph, J.J. (2005). Free-Marginal Multirater Kappa (multirater Kfree): Alternative to Fluiss’ Fixed-Marginal Multirater Kappa. Joensuu Learning and Instruction Symposium 2005, University of the Joensuu, Finland.

Sáenz-López, P., Ibáñez, S.J., Giménez, F.J., Sierra, A., \& Sánchez, M. (2005). Multifactor characteristic in the process of development of the male expert basketball player in Spain. International Journal of Sport Psychology, 36(2), 151-171.

Sáenz-López, P., Jiménez, A.C., Giménez, F.J., \& Ibáñez, S.J. (2007). La autopercepción de las jugadoras de baloncesto expertas respecto a sus procesos de formación. Cultura, Ciencia y Deporte, 7(3), 35-41.

Sgrò, F., Bracco, S., Pignato, S., \& Lipoma, M. (2018). Smallsided games and technical skills in soccer training: Systematic review and implications for sport and physical education practitioners. Journal of Sports Science, 6(1), 9-19. https://doi.org/10.17265/2332-7839/2018.01.002

Stølen, T., Chamari, K., Castagna, C., \& Wisløff, U. (2005). Physiology of soccer: an update. Sports Medicine, 35(6), 501-536.

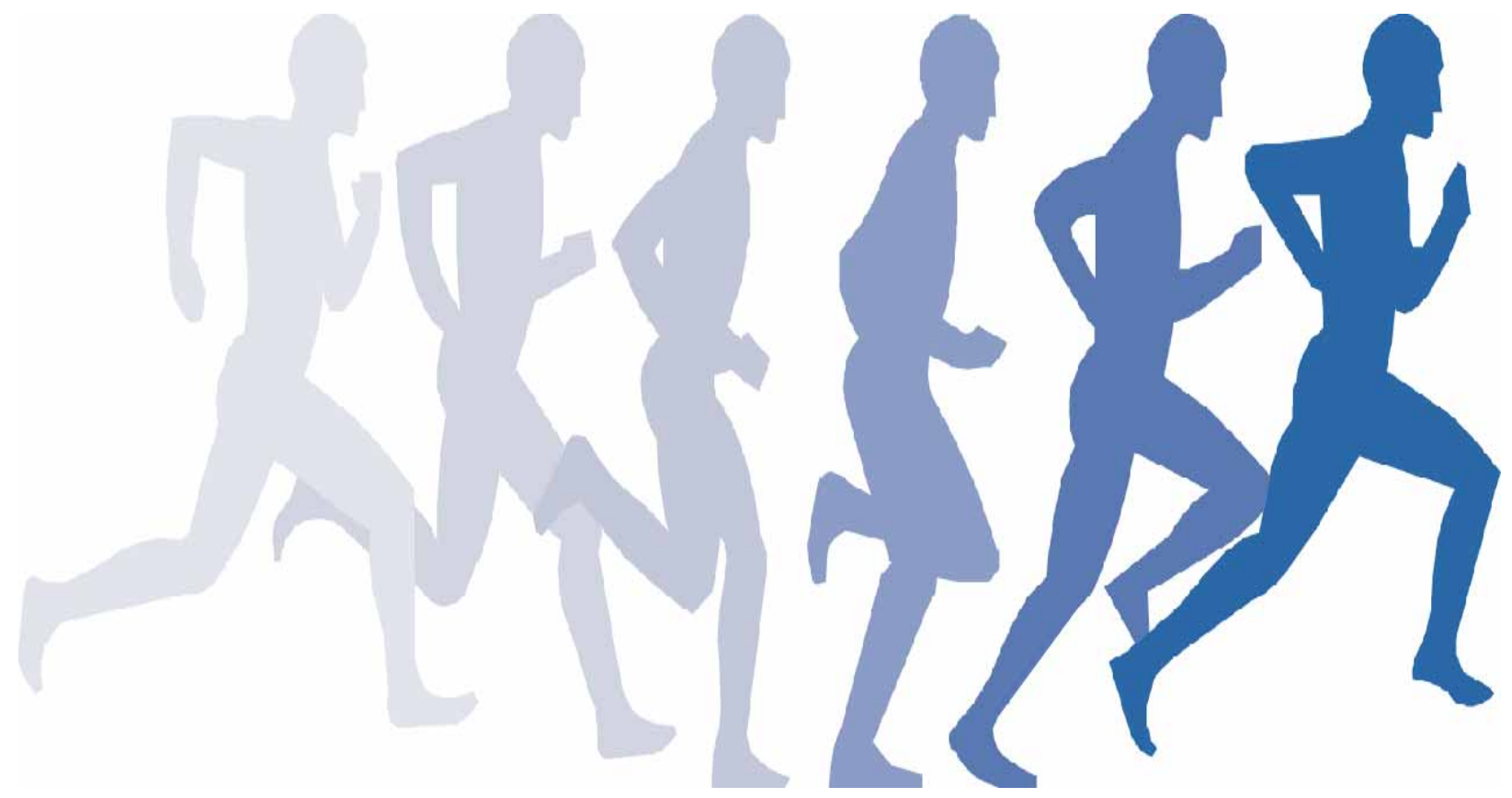

